\title{
Bistability in the Shape Transition of Strained Islands
}

\author{
H. J. W. Zandvliet ${ }^{1}$ and R. van Gastel ${ }^{2}$ \\ ${ }^{1}$ Physical Aspects of Nanoelectronics, MESA+ Institute for Nanotechnology, University of Twente, \\ P.O. Box 217, 7500 AE Enschede, The Netherlands \\ ${ }^{2}$ Solid State Physics, MESA+ Institute for Nanotechnology, University of Twente, P.O. Box 217, 7500 AE Enschede, The Netherlands
}

(Received 11 June 2007; published 28 September 2007)

\begin{abstract}
The equilibrium shape of a monatomic strained island on a substrate depends on the step free energies and the difference in surface stress between the island and the substrate. For small island sizes the step free energies dominate, resulting in compact islands. Beyond a critical island size, however, the strain energy becomes dominant and the island maximizes its perimeter, resulting in elongated islands. Here we show that for strained islands with force monopoles pointing in opposing directions at neighboring steps, a regime exists near the critical island size where both compact and elongated shapes can coexist.
\end{abstract}

DOI: 10.1103/PhysRevLett.99.136103

PACS numbers: 68.35.Md, 68.55.Jk

The synthesis of artificial low-dimensional structures has been a topic of scientific and technological interest for many years. The formation of two-dimensional quantum wells and zero-dimensional quantum dots using epitaxial growth techniques is well established. However, the preparation of one-dimensional nanowires turns out to be more complex. One attractive route is to grow wirelike structures at preexisting steps on a surface [1-3]. These wires are usually irregular because they meander along with the steps. Tersoff and Tromp [4] demonstrated that there is another route to form nanowires on a twodimensional substrate. They showed that strained islands that are formed during lattice-mismatched epitaxial growth, will initially have a compact shape governed by the ratio of the step free energies. Beyond a critical size the strain energy becomes the dominant factor and the island prefers to maximize its perimeter, resulting in extremely elongated epitaxial islands, i.e., nanowires [4-8]. In these heteroepitaxial systems the lattice mismatch between the island and the substrate leads to an elastic force monopole along the island periphery. For several metal adsorbates on $\mathrm{Si}(001)$, such as Ag [4], Bi [9], and Er [10], the spontaneous formation of nanowires has been reported.

Also in homoepitaxial systems, such as $\mathrm{Si} / \mathrm{Si}(001)$ and $\mathrm{Ge} / \mathrm{Ge}(001)$, an elastic force monopole can arise. In this case the origin of the force monopole is an anisotropy in the surface stress. In the specific case of the semiconductor group IV (001) surfaces, the dimerization of the surface leads to a compressive stress along the substrate dimer rows $\left(\sigma_{\|}\right)$and a tensile stress perpendicular to the dimer rows $\left(\sigma_{\perp}\right)[11,12]$. Because of the diamond crystal structure of $\mathrm{Si}$ and $\mathrm{Ge}$, the direction of the dimer rows rotates at each monatomic step. This rotation of the dimer rows introduces a force monopole $f= \pm\left(\sigma_{\|}-\sigma_{\perp}\right)$ at single layer height steps. The symmetry of the diamond lattice dictates that those force monopoles have to be pointing inwards for one step orientation and outwards for the perpendicular orientation.
Despite the fact that the energetics of the equilibrium shape as a function of the island size is in theory well understood and also experimentally well characterized, the actual shape transition of the structures from compact to elongated, or vice versa, has always been assumed to be a smooth transition. In this Letter we demonstrate that this assumption is not valid when force monopoles acting on neighboring boundaries point in opposing directions. Even though this configuration also exhibits a shape transition from a compact shape at small island sizes to elongated shapes at large island sizes [4-6], we will show that it is considerably more complex and significant differences occur between this configuration and the smooth shape transition that occurs when all force monopoles are pointing in the same direction. Although we will restrict ourselves here to islands of monatomic height, our results are also applicable to three-dimensional islands [4].

Consider a biaxially strained epitaxial island on a substrate with a twofold symmetry. For the sake of simplicity, we assume that the island (or vacancy island [13]) has a rectangular shape with length $l$ and width $w$, see Fig. 1. The island aspect ratio is $c^{2}=\frac{l}{w}$. The lattice mismatch between the island and the substrate (heteroepitaxy) or the surface stress anisotropy (homoepitaxy) introduces an elastic force monopole at the island boundaries. We denote the step free energy along the island periphery by $E_{l}$ and $E_{w}$, respectively. The total step free energy for the rectangular island is then

$$
E_{\text {step }}=2 l E_{l}+2 w E_{w}
$$

For the lattice-mismatched system the force monopole is generally constant along the island boundary and represented by $f$, whereas for the semiconductor group IV (001) surfaces one has $f_{l}=-f_{w}$. The island strain energy is calculated as [14]

$$
E_{\text {strain }}=-\frac{1}{2} \iint u\left[r_{1}, f\left(r_{2}\right)\right] f\left(r_{1}\right) d r_{1} d r_{2},
$$


where $u\left[r_{1}, f\left(r_{2}\right)\right]$ is the displacement at $r_{1}$ induced by a force $f$ at $r_{2}$. Li, Liu, and Lagally [5] have performed the integration of Eq. (2) for a rectangular island of length $l$ and width $w$. They showed that the total free energy per unit area, $E_{\text {tot }}\left(=\frac{E_{\text {step }}+E_{\text {strain }}}{l w}\right)$, can be written as

$$
E_{\mathrm{tot}}=\frac{2 E_{l}}{w}+\frac{2 E_{w}}{l}+\frac{2(l+w)}{l w} E_{s}\left[G(c)-2(1-\nu) \ln \frac{D}{a_{0}}\right],
$$

where $\mu$ is the bulk modulus, $\nu$ is Poisson's ratio, $a_{0}$ is a microscopic cutoff length, $E_{s}=\frac{1+\nu}{2 \pi \mu} f^{2}$ is the unit strain energy, $D^{2}=l w$ is the island size, and $G(c)$ is a dimensionless geometric factor. The geometric factor depends on the exact orientation of the force monopoles at the island boundaries. Li, Liu, and Lagally [5] have derived expressions for the case where the force monopoles have the same orientation at all island boundaries (referred to as case I). In the case that the force monopoles have opposing directions at neighboring boundaries (referred to as case II), a slightly different geometric factor was obtained [5].

$$
\begin{aligned}
G(c)= & \frac{1}{2\left(c+\frac{1}{c}\right)}\left\{2(1-\nu)\left[c \ln \left(\frac{\sqrt{c^{2}+\frac{1}{c^{2}}}+c}{\sqrt{c^{2}+\frac{1}{c^{2}}}-c}\right)+\frac{1}{c} \ln \left(\frac{\sqrt{c^{2}+\frac{1}{c^{2}}}+\frac{1}{c}}{\sqrt{c^{2}+\frac{1}{c^{2}}}-\frac{1}{c}}\right)-2 c \ln \left(\frac{c}{e}\right)-\frac{2}{c} \ln \left(\frac{1}{c e}\right)\right]\right. \\
& \left.+4\left[(1-4 \nu)\left(c+\frac{1}{c}\right)-2(1-3 \nu) \sqrt{c^{2}+\frac{1}{c^{2}}}\right]\right\}
\end{aligned}
$$

As in case I, in case II the equilibrium shape is compact for small islands, but beyond a critical island size the shape becomes elongated. Despite the great similarity in equations describing the geometric factor for both cases I and II and despite the fact that both geometric factors lead to a shape transition from compact to elongated, there is also a salient difference between the two cases. If one plots $E_{\text {tot }}$ versus the island aspect ratio $c^{2}$ for various island sizes $D^{2}$, as in case I one finds in case II a well-defined minimum at $c=1$ for small island sizes, see Fig. 2(a). In case I, a

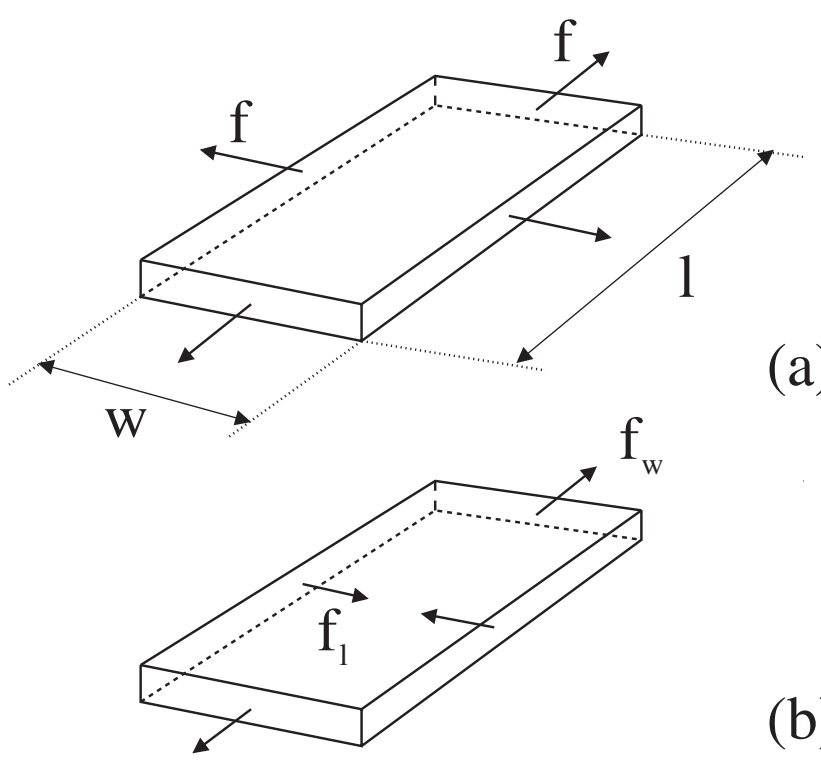

FIG. 1. Schematic drawing of a 2D island with length $l$ and width $w$ on a surface with twofold symmetry. (a) The elastic force monopoles along the island periphery are induced by a lattice mismatch (case I), (b) the elastic force monopoles along the island periphery are induced by an anisotropy in surface stresses (case II). smooth transition is found towards two new minima at $c^{2}$ and $\frac{1}{c^{2}}$, respectively. With increasing island size, these two minima gradually move away from $c=1$. In case II, however, just below the critical island size two additional local minima located at $c_{*}^{2}$ and $\frac{1}{c_{*}^{2}}$ develop (with $c_{*}>1$ ). With increasing island size these two local minima become deeper and deeper, whereas the minimum at $c=1$ becomes less pronounced, see Fig. 2(b). Eventually, all three minima have an equal depth and this point marks the transition from compact to elongated. For larger island sizes, the minimum at $c=1$ eventually vanishes, see Fig. 2(c).

Our evaluation of the island energy thus shows that the equilibrium shape of an island not only depends on the relative strengths of the step free energies and the strain energy, it is also a function of the mutual orientation of the force monopoles. The fact that case II exhibits a coexistence regime and case I does not, can be ascribed to the interaction between force monopoles at neighboring island boundaries. Changing the orientation of two of the four monopoles to point inwards, reorients them in such a way that the force monopoles are now pointing along the displacement that is caused by the force monopoles working on neighboring boundaries. This constitutes an additional gain in elastic energy at the island boundaries. For identical values of the step free energy, the additional gain in total energy per unit area of the island is given by

$$
\Delta E_{\mathrm{tot}}=8 \nu \frac{1+\nu}{\pi \mu} f^{2}\left(\frac{\sqrt{l^{2}+w^{2}}-(l+w)}{l w}\right) .
$$

Regardless of the island aspect ratio, a change in orientation of two of the four force monopoles will thus always lower the island free energy. The effect is strongest for aspect ratios $c \neq 1$ and the different orientation of the force monopoles thus makes the compact shape metastable 

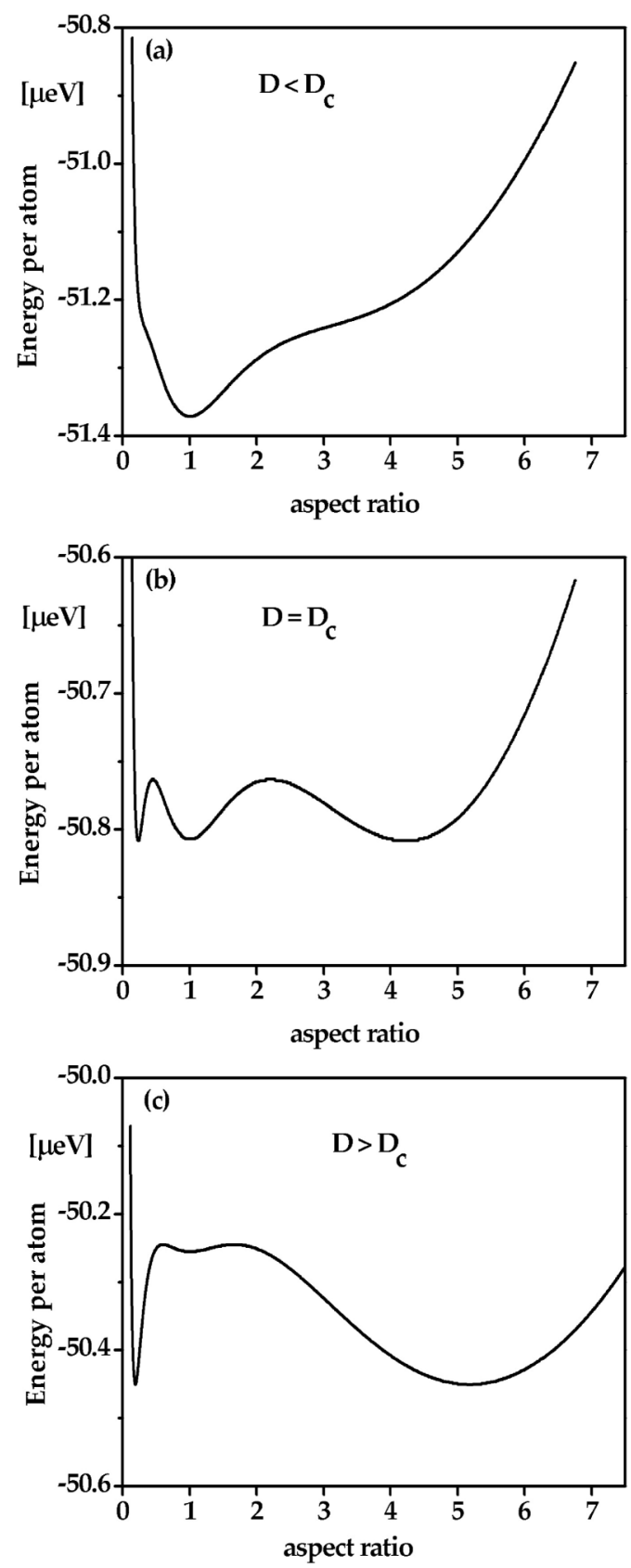

FIG. 2. Plot of the energy per atom $E_{\text {tot }}$ versus the island aspect ratio $c^{2}$ at various island sizes near the critical island size $D_{c}^{2}$ for case II. (a) $D<D_{c}, D^{2}=10816$ atoms (b) $D=D_{c}, D^{2}=$ 11342 atoms, and (c) $D>D_{c}, D^{2}=11881$ atoms.

or even unstable for island areas $D \approx D_{c}$. The spatial origin of the additional energy gain is at the corners of the island. Equation (5) is optimized by maximizing the amount of step length that is available directly adjacent to the corner sites. This is realized in practice through the premature elongation of the shape of the island at island areas $D<D_{C}$. The additional step length decreases $\Delta E_{\text {tot }}$ by an amount that makes it favorable for the island to assume an elongated shape, where the shape would otherwise still be compact, as in case I.
The evaluation of the island energy that is plotted in Fig. 2, shows the energy landscape for the case where the step energies $E_{l}$ and $E_{w}$ are equal. The introduction of an anisotropy in the step free energy with $\frac{E_{w}}{E_{l}}>1$ lowers the minimum at $c_{*}$ with respect to the one at $\frac{1}{c_{*}}$. Despite this difference, bistability is found here too. However, for low strain energies a regime exists where the bistability is suppressed as the $E_{w}$ boundary energy term in Eq. (3) becomes dominant over the additional gain in elastic energy. This effect is illustrated in Fig. 3, where we have calculated the evolution of $l$ and $w$ as a function of the island size for a small and a large strain energy. In Fig. 3 the bistability in the shape transition shows up as a discontinuity in the two branches that give $l$ and $w$ as a function of island area.

In practice, step free energies will decrease with increasing temperature until they vanish at the roughening temperature. The ratio $\frac{E_{s}}{E_{l}}$ can thus be made arbitrarily large and we therefore anticipate the bistability to occur in systems such as $\mathrm{Si}(001)$ and $\mathrm{Ge}(001)$, provided that the terrace size is sufficiently large. We have illustrated this by plotting the expected values for $\frac{E_{s}}{E_{l}}$ for $\mathrm{Ge}(001)$ in Fig. 4 versus the ratio $\frac{E_{w}}{E_{l}} . E_{w}$ and $E_{l}$ are determined from previously performed
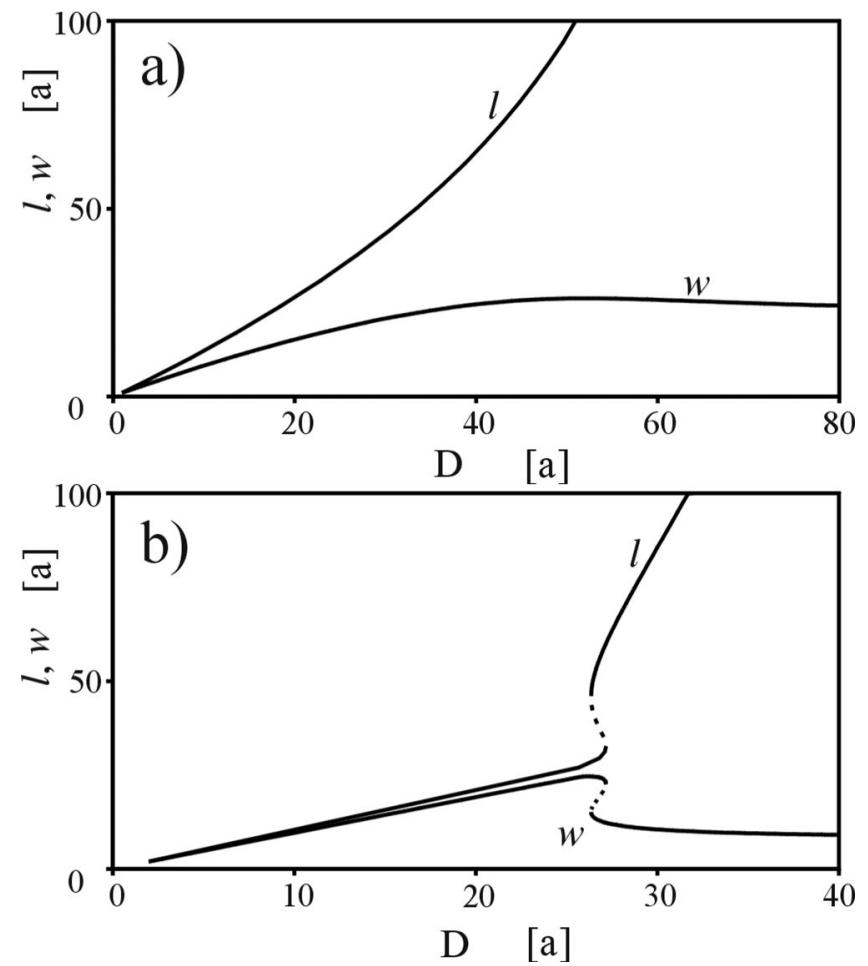

FIG. 3. A plot of the length $l$ and width $w$ of a strained island as a function of island area. The shape transition is clearly visible through the bifurcation of $l$ and $w$ into two distinct branches. The bistability affects the region $D \approx D_{c}$ where a hysteresis loop can be observed in the dashed section of the curve in panel (b). Energy values used to obtain this plot are $E_{l}=12 \mathrm{meV} / a$, $E_{w}=24 \mathrm{meV} / a$. (a) $\frac{E_{s}}{E_{l}}=\frac{2}{3}$ and (b) $\frac{E_{s}}{E_{l}}=66$. 


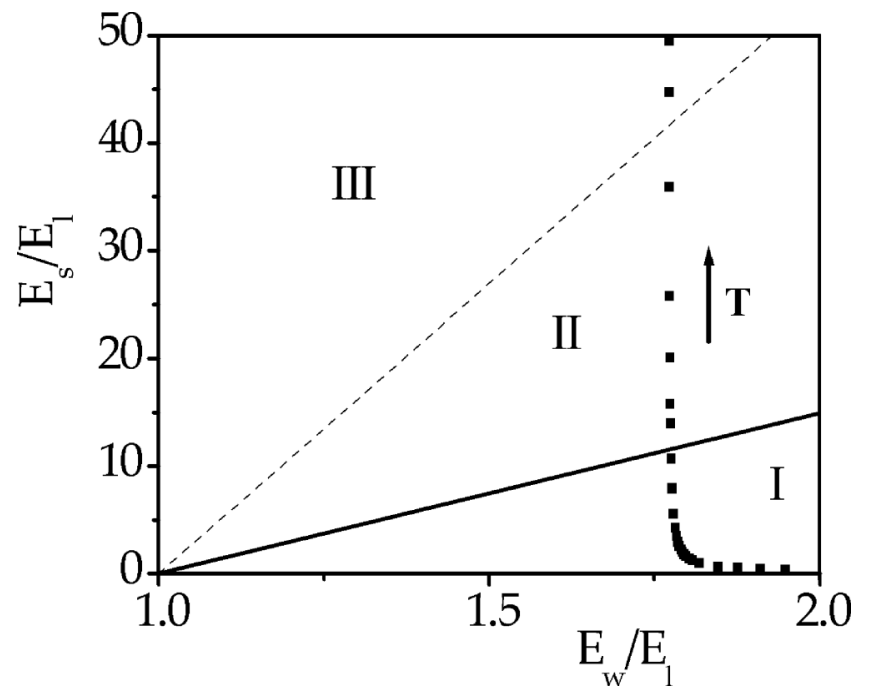

FIG. 4. Phase diagram of the ratio of the unit strain energy and the step free energy $\frac{E_{s}}{E_{l}}$ versus the step free energy ratio $\frac{E_{w}}{E_{l}}$. In region I the islands are always elongated, similar to the suppression of the shape transition under the influence of a stress anisotropy, as observed in Ref. [6]. In region II compact and elongated islands coexist near $D_{c}$, with the restriction that the long axis is always along the direction of the lowest step free energy. Finally, in region III compact and elongated islands coexist along both high symmetry axes near $D_{c}$. The data points (squares) refer to the values $\frac{E_{s}}{E_{l}}$ and $\frac{E_{w}}{E_{l}}$ of the $\mathrm{Ge}(001)$ surface at various temperatures, see Ref. [15] ( $f$ was taken as $\left.1 \mathrm{eV} / a^{2}\right)$. For $\mathrm{Ge}(001)$, the transition temperatures from region I to II and from region II to III are 885 and $894 \mathrm{~K}$, respectively.

STM measurements of the step free energies on $\mathrm{Ge}(001)$ [15]. With increasing ratio $\frac{E_{w}}{E_{l}}$ the bistability is suppressed more and more and the temperature interval at which the bistability can be observed narrows. For Ge(001), the crossover point from the region with exclusively elongated islands into the regime where a bistability occurs, lies at $885 \mathrm{~K}$, i.e., approximately $15 \mathrm{~K}$ below the roughening temperature. We therefore conclude that the bistability that we have found here, will occur in practice in the semiconductor group IV (001) surfaces. Its observation can, however, be hampered by the fact that it occurs only at elevated temperatures, just below the roughening temperature. It should be pointed out here that for less anisotropic step energies, the bistability occurs over a much larger temperature interval. In the case of fully isotropic step energies, the bistability is found for all nonzero values of the strain energy. The minimum size of the terraces for which the effect should be observable is at least a few times the critical diameter $D_{c}$.

A second fundamental conclusion of our calculations is that the precise shape of a strained island can depend critically on how it was formed. Depending on whether one approaches the critical island size from below or above, i.e., through growth or decay, in case II the island shape is temporarily trapped in the compact shape $(c=1)$ or in the elongated shape $\left(c_{*}\right)$, respectively. This is illustrated in Fig. 3(b), which demonstrates a discontinuous jump in $l$ and $w$ as the island size is changed and the island shape is trapped on either the compact branch or the elongated branch. We speculate that a similar effect can be found for three-dimensional islands when an approach similar to that in Ref. [4] is applied.

In summary, we have shown that strained islands on a surface with twofold symmetry undergo a shape transition from compact to elongated via a coexistence regime. The coexistence regime occurs when the force monopoles at the island boundaries change direction at each neighboring boundary, although their relative magnitude may vary. The applicability of the theory is not restricted to homoepitaxial systems only. In principle it applies to heteroepitaxial systems as well, provided that the force monopoles at neighboring boundaries point in opposite directions.

[1] J. R. Ahn, P. G. Kang, K. D. Ryang, and H. W. Yeom, Phys. Rev. Lett. 95, 196402 (2005).

[2] P. Segovia, D. Purdie, M. Hengsberger, and Y. Baer, Nature (London) 402, 504 (1999).

[3] P. Gambardella, A. Dallmeyer, K. Maiti, M. C. Malagoli, W. Eberhardt, K. Kern, and C. Carbone, Nature (London) 416, 301 (2002).

[4] J. Tersoff and R. M. Tromp, Phys. Rev. Lett. 70, 2782 (1993).

[5] A. Li, F. Liu, and M. G. Lagally, Phys. Rev. Lett. 85, 1922 (2000).

[6] A. Pradhan, N.-Y. Ma, and F. Liu, Phys. Rev. B 70, 193405 (2004).

[7] V. Zielasek, F. Liu, Y. Zhao, J. Maxson, and M. Lagally, Phys. Rev. B 64, 201320(R) (2001).

[8] R. van Gastel, N. C. Bartelt, and G. L. Kellogg, Phys. Rev. Lett. 96, 036106 (2006).

[9] K. Miki, J. H. G. Owen, D. R. Bowler, G. A. D. Briggs, and K. Sakamoto, Surf. Sci. 421, 397 (1999); see also K. Miki, D. R. Bowler, J.H.G. Owen, G. A.D. Briggs, and K. Sakamoto, Phys. Rev. B 59, 14868 (1999).

[10] Y. Chen, D. A. A. Ohlberg, G. Medeiros-Riberio, Y. A. Chang, and R.S. Williams, Appl. Phys. Lett. 76, 4004 (2000).

[11] H. J. W. Zandvliet, Rev. Mod. Phys. 72, 593 (2000).

[12] H. J. W. Zandvliet, Phys. Rep. 388, 1 (2003).

[13] M. T. Middel, H. J. W. Zandvliet, and B. Poelsema, Phys. Rev. Lett. 88, 196105 (2002).

[14] L. D. Landau and E. M. Lifshitz, Theory of Elasticity (Pergamon Press, New York, 1959).

[15] H. J. W. Zandvliet, Phys. Rev. B 61, 9972 (2000). 